Korean J. Math. 20 (2012), No. 1, pp. 107-116

\title{
EXISTENCE OF THE SOLUTIONS FOR THE SINGULAR POTENTIAL ELLIPTIC SYSTEM
}

\author{
Tacksun Jung And Q-Heung Choi*
}

\begin{abstract}
We investigate the multiple solutions for a class of the elliptic system with the singular potential nonlinearity. We obtain a theorem which shows the existence of the solution for a class of the elliptic system with singular potential nonlinearity and Dirichlet boundary condition. We obtain this result by using variational method and critical point theory.
\end{abstract}

\section{Introduction and statement of main result}

Let $\Omega$ be a bounded subset of $R^{n}$ with smooth boundary. Let $D$ be an open subset in $R^{n}$ with compact complement $C=R^{n} \backslash D, n \geq 2$. In this paper we investigate the multiple solutions $U(\cdot) \in C^{2}(\Omega, D)$ for a class of the elliptic system with the singular potential nonlinearity and Dirichlet boundary condition

$$
\begin{aligned}
\Delta U(x) & =\operatorname{grad}_{U} G(x, U(x)) \quad \text { in } \quad \Omega, \\
U & =(0, \cdots, 0) \quad \text { on } \partial \Omega
\end{aligned}
$$

where $G \in C^{2}\left(\Omega \times D, R^{1}\right)$ and $U=\left(u_{1}, \ldots, u_{n}\right)$. We assume that $G$ satisfies the following conditions:

Received January 19, 2012. Revised March 12, 2012. Accepted March 15, 2012. 2010 Mathematics Subject Classification: 34C15, 34C25, 35Q72.

Key words and phrases: Elliptic system, singular potential nonlinearity, Dirichlet boundary condition, variational method, critical point theory, deformation retract, $(P . S .)_{c}$ condition.

This work (Tacksun Jung) was supported by Basic Science Research Program through the National Research Foundation of Korea (NRF) funded by the Ministry of Education, Science and Technology (KRF-2011-0026920).

${ }^{*}$ Corresponding author. 
(G1) There exists $R_{0}>0$ such that

$$
\sup \left\{|G(x, U)|+\left\|\operatorname{grad}_{U} G(x, U)\right\|_{R^{n}} \mid(x, U) \in \Omega \times\left(R^{n} \backslash B_{R_{0}}\right)\right\}<+\infty .
$$

(G2) There is a neighborhood $Z$ of $C$ in $R^{n}$ such that

$$
G(x, U) \geq \frac{A}{d^{2}(U, C)} \text { for } \quad(x, U) \in \Omega \times Z,
$$

where $d(U, C)$ is the distance function to $C$ and $A>0$ is a constant. Let $U=\left(u_{1}, \ldots, u_{n}\right)$. The system (1.1) can be rewritten as

$$
\begin{aligned}
\delta u_{1}(x)= & \frac{\partial}{\partial u_{1}} g(x, u(x)) \quad \text { in } \omega, \\
\delta u_{2}(x)= & \frac{\partial}{\partial u_{2}} g(x, u(x)) \quad \text { in } \omega, \\
& \vdots \quad \vdots \quad \vdots,
\end{aligned}
$$

where $\operatorname{grad}_{U} G(x, U(x))=\left(G_{u_{1}}(x, U), \ldots, G_{u_{n}}(x, U)\right)$. Let $0<\lambda_{1}<$ $\lambda_{2} \leq \ldots \leq \lambda_{k} \leq \ldots$ be the eigenvalues and $\phi_{k}$ be the eigenfunctions belonging to the eigenvalue $\lambda_{k}, k \geq 1$, of the eigenvalue problem for a single elliptic problem

$$
\begin{aligned}
-\Delta u & =\lambda u \quad \text { in } \quad \Omega, \\
u & =0 \quad \text { on } \quad \partial \Omega .
\end{aligned}
$$

We note that $\phi_{1}(x)$ is the positive normalized eigenfunction associated to $\lambda_{1}$. Let $H=H_{0}^{1}\left(\Omega, R^{n}\right)$. We endow the Hilbert space $H$ with the norm

$$
\|U\|_{H}^{2}=\sum_{i=1}^{n}\left\|u_{i}\right\|^{2},
$$

where $\left\|u_{i}\right\|^{2}=\int_{\Omega}\left|\nabla u_{i}(x)\right|^{2} d x$. Thus we have

$$
\|U\|_{H}=\int_{\Omega}\|\nabla U(x)\|_{R^{n}}^{2} .
$$


In this paper we are trying to find the weak solutions $U \in C^{2}(\Omega, D) \cap H$ of the system (1.1), that is, $U=\left(u_{1} \ldots, u_{n}\right) \in C^{2}(\Omega, D) \cap H$ such that

$$
\begin{gathered}
\int_{\Omega}\left[\nabla u_{1} \cdot \nabla \phi_{1}+\nabla u_{2} \cdot \nabla \phi_{2}+\cdots+\nabla u_{n} \cdot \nabla \phi_{n}\right] d x+\int_{\Omega} \frac{\partial}{\partial u_{1}} G(x, U(x)) \cdot \phi_{1} \\
+\int_{\Omega} \frac{\partial}{\partial u_{2}} G(x, U(x)) \cdot \phi_{2}+\cdots+\int_{\Omega} \frac{\partial}{\partial u_{n}} G(x, U(x)) \cdot \phi_{n}=0,
\end{gathered}
$$

for all $\phi=\left(\phi_{1}, \cdots, \phi_{n}\right) \in C^{2}(\Omega, D) \cap H$, i.e.,

$$
\int_{\Omega}[\nabla U \cdot \nabla \phi] d x+\int_{\Omega} \operatorname{grad}_{U} G(x, U(x)) \cdot \phi=0 \text {, for all } \phi \in C^{2}(\Omega, D) \cap H \text {. }
$$

In [1-7] the authors investigate the existence of multiple solution of elliptic problems. In [8] there are many methods to study the existence of multiple solution of elliptic problems and some simple nonlinear problems. Our main result is the following:

Theorem 1.1. Assume that $G$ satisfies the conditions $(G 1)-(G 2)$. Then system (1.1) has at least one solution.

For the proof of Theorem 1.1 we approach the variational method and the critical point theory. In section 2, we investigate the (P.S.) condition for the associated functional of (1.1). In section 3, we prove Theorem 1.1 by the some variant of the mountain pass theorem in critical point theory.

\section{Palais Smale Condition}

Since $\left|\lambda_{i}\right| \geq 1$ for all $i \geq 1$, we have the following lemma.

Lemma 2.1. Let $u \in H_{0}^{1}(\Omega, R)$ and $\|\cdot\|$ is a Sobolev norm. Then

(i) $\|u\| \geq C\|u\|_{L^{2}(\Omega)}$ for some constant $C>0$.

(ii) $\|u\|=0$ if and only if $\|u\|_{L^{2}(\Omega)}=0$.

(iii) $-\Delta u \in W_{0}^{1,2}(\Omega, R)$ implies $u \in W_{0}^{1,2}(\Omega, R)$.

Proof. (i) and (ii) can be checked easily.

(iii) Let $\lambda_{n}$ be an eigenvalue of the eigenvalue problem for a single elliptic equation $-\Delta u=\lambda u$ in $\Omega$ with Dirichlet boundary condition. We note that $\left\{\lambda_{n}:\left|\lambda_{n}\right|<|c|\right\}$ is finite. Let us set $f=-\Delta u \in W_{0}^{1,2}(\Omega, R)$. Then $f$ can be expressed by

$$
f=\sum h_{n} \phi_{n} .
$$


Then

Hence we have the inequality

$$
(-\Delta)^{-1} f=\sum \frac{1}{\lambda_{n}} h_{n} \phi_{n}
$$

$$
\left\|(-\Delta)^{-1} f\right\|^{2}=\sum \lambda_{n}^{2} \frac{1}{\lambda_{n}^{2}} h_{n}^{2} \leq \sum h_{n}^{2}
$$

which means that

$$
\left\|(-\Delta)^{-1} f\right\| \leq\|f\|_{L^{2}(\Omega)}
$$

Let us introduce an open set of the Hilbert space $H_{0}^{1}\left(\Omega, R^{n}\right)$ as follows

$$
E=\left\{U \in H_{0}^{1}\left(\Omega, R^{n}\right) \mid U(x) \in D \subset R^{n}, x \in \Omega\right\} .
$$

Let us define the functional on $E$

$$
I(U)=\int_{\Omega}\left(\frac{1}{2}\|\nabla U(x)\|_{R^{n}}^{2}+G(x, U(x))\right) d x,
$$

where $\|U\|_{H}^{2}=\int_{\Omega}\|\nabla U\|_{R^{n}}^{2}=\sum_{i=1}^{n}\left\|\nabla u_{i}\right\|_{R^{1}}^{2}$. The Euler equation for (2.1) is (1.1). By the following proposition 2.1, $I \in C^{1}(E, R)$, and so the weak solutions of system (1.1) coincide with the critical points of the associated functional $I(U)$.

Proposition 2.1. Assume that $G$ satisfies the conditions $(G 1)-$ $(G 2)$. Then $I(U)$ is continuous and Fréchet differentiable in $E$ with Fréchet derivative

$$
\begin{aligned}
& D I(U) V \\
& \quad=\int_{\Omega}\left((-\Delta U(x)) \cdot V(x)+\operatorname{grad}_{U} G(x, U(x)) \cdot V(x)\right) d x, \quad \forall V \in E .
\end{aligned}
$$

Moreover $D I \in C$. That is, $I \in C^{1}$.

Proof. First we prove that $I(U)$ is continuous. For $U, V \in E$,

$$
\begin{aligned}
& \left.|I(U+V)-I(U)|=\mid \frac{1}{2} \int_{\Omega}(-\Delta U(x)-\Delta V(x))\right) \cdot(U(x)+V(x)) d x \\
& +\int_{\Omega} G(x, U(x)+V(x)) d x-\frac{1}{2} \int_{\Omega}(-\Delta U(x)) \cdot U(x) d x-\int_{\Omega} G(x, U(x)) d x \\
& =\mid \frac{1}{2} \int_{\Omega}\left[(-\Delta U \cdot V-\Delta V \cdot U-\Delta V \cdot V) d x+\int_{\Omega}(G(x, U+V)-G(x, U)) d x \mid .\right.
\end{aligned}
$$


We have

$$
\begin{aligned}
& \left|\int_{\Omega}[G(x, U+V)-G(x, U)] d x\right| \\
& \leq\left|\int_{\Omega}\left[\operatorname{grad}_{U} G(x, U(x)) \cdot V+O\left(\|V\|_{R^{n}}\right)\right] d x\right| \\
& =O\left(\|V\|_{R^{n}}\right) .
\end{aligned}
$$

Thus we have

$$
|I(U+V)-I(U)|=O\left(\|V\|_{R^{n}}\right)
$$

Next we shall prove that $I(U)$ is Fréchet differentiable in $E$. For $U, V \in$ $E$,

$$
\begin{aligned}
|I(U+V)-I(U)-\nabla I(U) V| & \mid \frac{1}{2} \int_{\Omega}(-\Delta U-\Delta V) \cdot(U+V) d x+\int_{\Omega} G(x, U+V) d x-\frac{1}{2} \int_{\Omega}(-\Delta U) \cdot U d x \\
& -\int_{\Omega} G(x, U) d x-\int_{\Omega}\left(-\Delta U+\operatorname{grad}_{U} G(x, U(x))\right) \cdot V d x \mid \\
= & \mid \frac{1}{2} \int_{\Omega}[-\Delta U \cdot V-\Delta V \cdot U-\Delta V \cdot V] d x+\int_{\Omega}[G(x, U+V)-G(x, U)] d x \\
& -\int_{\Omega}\left[\left(-\Delta U+\operatorname{grad}_{U} G(x, U(x))\right) \cdot V\right] d x \mid .
\end{aligned}
$$

Thus by (2.3), we have

$$
|I(U+V)-I(U)-D I(U) V|=O\left(\|V\|_{R^{n}}\right) .
$$

Similarly, it is easily checked that $I \in C^{1}$.

Lemma 2.2. Assume that $G$ satisfies the conditions $(G 1)-(G 2)$. Let $\left\{U_{k}\right\} \subset E$ and $U_{k} \rightarrow U$ weakly in $E$ with $U \in \partial E$. Then $I\left(U_{k}\right) \rightarrow \infty$.

Proof. To prove the conclusion, it suffices to prove that

$$
\int_{\Omega} G\left(x, U_{k}(x)\right) d x \longrightarrow+\infty .
$$

Since $G(x, U(x))$ is bounded from below, it suffices to prove that there is a subset $\tilde{\Omega}$ of $\Omega$ such that

$$
\int_{\tilde{\Omega}} G\left(x, U_{k}(x)\right) d x \longrightarrow+\infty .
$$

$U \in \partial E$ means that there exists $x^{*} \in \Omega$ such that $U\left(x^{*}\right) \in \partial D$. Let us set

$$
\Omega_{\delta}\left(x^{*}\right)=\left\{x \in \Omega \mid\left\|x-x^{*}\right\|_{R^{n}}<\delta\right\} .
$$


By $(G 1)$ and $(G 2)$, there exists a constant $B$ such that

$$
G(x, U) \geq \frac{A}{d^{2}(U, C)}-B .
$$

Thus we have

$$
\int_{\Omega_{\delta}\left(x^{*}\right)} G(x, U(x)) d x \geq \int_{\Omega_{\delta}\left(x^{*}\right)}\left(\frac{A}{\left\|U(x)-U\left(x^{*}\right)\right\|_{R^{n}}^{2}}-B\right) d x
$$

for all $\delta>0$. By Schwarz's inequality, we have

$$
\left\|U(x)-U\left(x^{*}\right)\right\|_{R^{n}} \leq\left\|x-x^{*}\right\|_{R^{n}}^{\frac{1}{2}}\left(\int_{\Omega}\|\nabla U(x)\|_{R^{n}}^{2}\right)^{\frac{1}{2}} \leq \delta^{\frac{1}{2}}\left(\int_{\Omega}\|\nabla U(x)\|_{R^{n}}^{2}\right)^{\frac{1}{2}} .
$$

Thus we have

$$
\int_{\Omega_{\delta}\left(x^{*}\right)} G(x, U(x)) d x \geq \int_{\Omega_{\delta}\left(x^{*}\right)}\left(\frac{A}{\delta\|U\|_{H}^{2}}-B\right) d x \longrightarrow \infty .
$$

Hence

$$
\int_{\Omega_{\delta}\left(x^{*}\right)} G(x, U(x)) d x=\infty .
$$

Since the embedding $H \hookrightarrow C\left(\Omega, R^{n}\right)$ is compact, we have

$$
\max \left\{\left\|U(x)-U_{k}(x)\right\|_{R^{n}}^{2} \mid x \in \Omega\right\} \longrightarrow 0 \quad \text { as } k \rightarrow \infty .
$$

Thus by Fatou's lemma, we have

$$
\begin{gathered}
\liminf \int_{G_{\delta}\left(x^{*}\right)} G\left(x, U_{k}(x)\right) \geq \int_{G_{\delta}\left(x^{*}\right)} \liminf G\left(x, U_{k}(x)\right) \\
=\int_{G_{\delta}\left(x^{*}\right)} G(x, U(x))=+\infty .
\end{gathered}
$$

Thus

$$
\liminf \int_{G_{\delta}\left(x^{*}\right)} G\left(x, U_{k}(x)\right)=+\infty .
$$

Thus

$$
I\left(U_{k}\right)=\int_{\Omega}\left[\frac{1}{2}\left\|\nabla U_{k}\right\|_{R^{n}}^{2}+G\left(x, U_{k}(x)\right)\right] d x \longrightarrow+\infty,
$$

so we prove the lemma.

Lemma 2.3. (Palais-Smale condition) Assume that $G$ satisfies the conditions $(G 1)$ and $(G 2)$. Then there exists a constant $\gamma_{0}$ depending on $C^{1}$ norm of the function $G$ on $\Omega \times\left(R^{n} \backslash B_{R_{0}}\right)$ such that $I(u)$ satisfies the $(P . S .)_{\gamma}$ condition for $\gamma>\gamma_{0}$. 
Proof. We shall prove the lemma by contradiction. We suppose that there exists a sequence $\left\{U_{k}\right\} \subset E$ satisfying $I\left(U_{k}\right) \rightarrow \gamma$ and

$$
D I\left(U_{k}\right)=-\Delta U_{k}+\operatorname{grad}_{U} G\left(x, U_{k}(x)\right) \longrightarrow \theta \quad \text { in } E,
$$

or equivalently

$$
U_{k}+(I-\Delta)^{-1}\left(\operatorname{grad}_{U} G\left(x, U_{k}(x)\right)-U_{k}\right) \longrightarrow \theta,
$$

where $\theta=(0, \cdots, 0)$ and $(I-\Delta)^{-1}$ is a compact operator. We claim that the sequence $\left\{U_{k}\right\}$, up to a subsequence, converges. Since $G$ is bounded below and

$$
I\left(U_{k}\right)=\int_{\Omega}\left[\frac{1}{2}\left\|\nabla U_{k}\right\|_{R^{n}}^{2}+G\left(x, U_{k}(x)\right)\right] d x \longrightarrow \gamma,
$$

there exists a constant $\tau>0$ such that

$$
\int_{\Omega}\left\|\nabla U_{k}\right\|_{R^{n}}^{2} d x \leq \tau .
$$

We shall prove that the sequence $\left\{U_{k}\right\}$, up to a subsequence, is bounded in $H_{0}^{1}\left(\Omega, R^{n}\right)$. If then, there is a subsequence, up to a subsequence, $U_{k}$ converging weakly to $U$ in $E$. By Lemma 2.2, we have that $U \in E$ and that $\left\|\operatorname{grad}_{U} G\left(\cdot, U_{k}\right)\right\|$ is bounded. Since $(I-\Delta)^{-1}$ is compact and (2.5) holds, $\left\{U_{k}\right\}$ converges strongly to $U$. Let $V_{k_{i}}=\frac{1}{|\Omega|} \int_{\Omega} U_{k_{i}}(x) d x, i=$ $1, \ldots, n$, where $U_{k}=\left(U_{k_{1}}, \cdots, U_{k_{n}}\right)$, and $V_{k}=\left(V_{k_{1}}, \cdots, V_{k_{n}}\right)$. If $\left\{V_{k}\right\}$ is bounded, then $\left\{U_{k}\right\}$ is bounded in $H_{0}^{1}\left(\Omega, R^{n}\right)$. Thus it suffices to prove that $\left\{V_{k}\right\}$ is bounded. By contradiction, we suppose that $\left\|V_{k}\right\|_{R^{n}} \rightarrow \infty$. Then for large $k$, we have

$$
\left\|U_{k}(x)\right\|_{R^{n}} \geq\left\|V_{k}\right\|_{R^{n}}-\left(|\Omega| \int_{\Omega}\left\|\nabla U_{k}\right\|_{R^{n}}^{2} d x\right)^{\frac{1}{2}} \geq R_{0} .
$$

It follows from (2.6) that

$$
\begin{aligned}
& \left|\int_{\Omega} G(x, U(x)) d x\right| \\
& \quad \leq|\Omega| \sup \left\{|G(x, U(x))| \mid(x, U(x)) \in \Omega \times\left(R^{n} \backslash B_{R_{0}}\right)\right\} .
\end{aligned}
$$

By (2.5), for large $k$, we have

$$
\int_{\Omega}\left[\left\|\nabla U_{k}(x)\right\|_{R^{n}}^{2}+\operatorname{grad}_{U} G\left(x, U_{k}(x)\right)\left(U_{k}-V_{k}\right)\right] d x \leq\left\|U_{k}-V_{k}\right\|_{H} .
$$

Since $\int_{\Omega}\left[U_{k}-V_{k}\right] d x=0$, we have

$$
\left\|\left(U_{k}-V_{k}\right)\right\|_{H}=\left\|\nabla U_{k}\right\|_{L^{2}}, \quad\left\|\left(U_{k}-V_{k}\right)\right\|_{L^{2}} \leq\left\|\left(U_{k}-V_{k}\right)\right\|_{H} .
$$


It follows that

$$
\int_{\Omega}\left\|\nabla U_{k}\right\|_{R^{n}}^{2} d x \leq\left\|\nabla U_{k}\right\|_{L^{2}}+\left\|\operatorname{grad}_{U} G\left(x, U_{k}\right)\right\|_{L^{2}}\left\|\nabla U_{k}\right\|_{L^{2}} .
$$

Thus we have

$$
\begin{aligned}
\left\|\nabla U_{k}\right\|_{L^{2}} & \leq 1+\left\|\operatorname{grad}_{U} G\left(x, U_{k}\right)\right\|_{L^{2}} \\
& \leq 1+|\Omega| \sup _{(x, U) \in \Omega \times\left(R^{n} \backslash B_{R_{0}}\right)}\left\|\operatorname{grad}_{U} G\left(x, U_{k}\right)\right\|_{R^{n}} .
\end{aligned}
$$

Let

$$
\begin{aligned}
\gamma_{0}=\frac{1}{2}(1 & \left.+|\Omega| \sup _{(x, U) \in \Omega \times\left(R^{n} \backslash B_{R_{0}}\right)}\left\|\operatorname{grad}_{U} G\left(x, U_{k}\right)\right\|_{R^{n}}\right)^{2} \\
& +|\Omega| \sup _{(x, U) \in \Omega \times\left(R^{n} \backslash B_{R_{0}}\right)}\left|G\left(x, U_{k}\right)\right| .
\end{aligned}
$$

Then by (2.7) and (2.8), $I\left(U_{k}\right) \leq \gamma_{0}$, which leads to a contradiction. Thus we prove the lemma.

\section{Proof of Theorem 1.1}

By Proposition 2.1, $I(U)$ is continuous and Fréchet differentiable in $E$ and moreover $D I \in C$. By Lemma 2.2, If $\left\{U_{k}\right\} \subset E$ and $U_{k} \rightarrow U$ weakly in $E$ with $U \in \partial E$, then $I\left(U_{k}\right) \rightarrow \infty$. By Lemma 2.3, there exists a constant $\gamma_{0}$ depending on $C^{1}$ norm of the function $G$ on $\Omega \times\left(R^{n} \backslash B_{R_{0}}\right)$ such that $I(u)$ satisfies the $(P . S .)_{\gamma}$ condition for $\gamma>\gamma_{0}$. Let us choose an element $U \in \partial E$ and a small neighborhood $B_{r}(U)$ of $U$ with radius $r>0$. We can choose an $U_{0} \in B_{r}(U) \cap E$. We also choose elements $U_{1}$ and $U_{2}$ such that $U_{1}, U_{2} \in E \backslash B_{r}(U)$. Let us define a class of sets as follows:

$$
\begin{aligned}
& \Gamma=\{K \subset E \mid K \text { is closed and connected, } \\
& \left.\qquad U_{0} \in B_{r}(U) \cap E, U \in \partial E, \quad U_{1}, U_{2} \in E \backslash B_{r}(U)\right\} .
\end{aligned}
$$

By Lemma 2.2, we can choose a small radius $r>0$ such that $I\left(U_{0}\right)>$ $I\left(U_{1}\right)$ and $I\left(U_{0}\right)>I\left(U_{2}\right)$, where $U_{0} \in B_{r}(U) \cap E, U_{1}, U_{2} \in E \backslash B_{r}(U)$. Let us set

$$
c=\inf _{K \in \Gamma} \max _{U \in K} I(U) .
$$


We know that by the mountain pass theorem, $c$ is the critical value of $I(u)$. Thus it remains to show that $c>\gamma_{0}$. Let us set

$$
C_{\gamma}=\{U \in E \mid I(U)<\gamma\} .
$$

Suppose that $c \leq \gamma_{0}$. Then there is a closed and connected set $K \in \Gamma$ containing three points $U_{0} \in B_{r}(U) \cap E, U \in \partial E$ and $U_{1}, U_{2} \in E \backslash B_{r}(U)$ such that $K \subset C_{\gamma_{0}+1}$. But we can choose a small number $r>0$ such that $U_{0} \in B_{r}(U) \cap E$ and $I\left(U_{0}\right)>\gamma_{0}+1$. Then

$$
\max _{U \in K} I(U) \geq \inf _{K \in \Gamma} \max _{U \in K}>\gamma_{0}+1,
$$

which is absurd to the assumption that $c \leq \gamma_{0}$. Thus $c>\gamma_{0}$. We prove the theorem.

\section{References}

[1] A. Castro and J. Cossio, Multiple Solutions for a nonlinear Dirichlet problem, SIAM J. Math. Anal. 25, (6) (1994), 1554-1561.

[2] A. Castro and A. C. Lazer, Critical point theory and the number of solutions of a nonlinear Dirichlet problem, Ann. Mat. Pura Appl. 120 (4) (1979), 113-137.

[3] M. Degiovanni, Homotopical properties of a class of nonsmooth functions, Ann. Mat. Pura Appl. 156 (1990), 37-71.

[4] A. Groli, A. Marino and C. Saccon, Variational theorems of mixed type and asymptotically linear variational inequalities, Topol. Methods Nonlinear Anal. 12 (1998), 109-136.

[5] K.S. Ha and Y.H. Lee, Existence of multiple positive solutions of singular boundary value problems, Nonlinear Anal. TMA, 28 (1997), 1429-1438.

[6] K. Lan and R.L. Webb, Positive solutions of semilinear equation with singularities, J. Differential Equations, 148 (1998), 407-421.

[7] A. M. Micheletti and A. Pistoia, Multiplicity results for a fourth-order semilinear elliptic problem, Nonlinear Anal. TMA, 31 (7) (1998), 895-908.

[8] P. H. Rabinowitz, Minimax methods in critical point theory with applications to differential equations, CBMS Reg. Conf. Ser. in Math. 6, Amer. Math. Soc., Providence, RI, 1986.

Department of Mathematics

Kunsan National University

Kunsan 573-701, Korea

E-mail: tsjung@kunsan.ac.kr 
Department of Mathematics Education

Inha University

Incheon 402-751, Korea

E-mail: qheung@inha.ac.kr 